weeks, with flattening of the lesions on the glans. All lesions were noted by the patient to have cleared after four weeks of imiquimod use, and only post inflammatory hyperpigmentation was noted at his review after six weeks. Minimal side effects were noted by the patient except for transient itch.

Discussion We report our first case of penile bowenoid papulosis responding to imiquimod monotherapy, and is the 5 th reported case to date. Our case demonstrates one of the most rapid clinical clearance within six weeks, after only four weeks of imiquimod application. Other treatment modalities like electrocurrettage, 5 -fluorouracil or topical interferon have all been associated with recurrence. Immunomodulatory treatment for this condition appears safe and efficacious, with the added convenience of being patient administered.

\section{P2.085 THE CURRENT ANTIMICROBIAL SUSCEPTIBILITY IN CHLAMYDIA TRACHOMATIS IN JAPAN FROM THE NATIONWIDE SURVEILLANCE}

doi:10.1136/sextrans-2013-051184.0349

\begin{abstract}
1,2S Takahashi, ${ }^{2} \mathrm{~T}$ Matsumoto, ${ }^{2} \mathrm{~S}$ Yamamoto, ${ }^{2} \mathrm{~K}$ Ishikawa, ${ }^{2} \mathrm{~S}$ Uehara, ${ }^{2} \mathrm{R}$ Hamasuna, ${ }^{2} \mathrm{M}$ Yasuda, ${ }^{2} \mathrm{H}$ Hayami, ${ }^{3} \mathrm{H}$ Hanaki, ${ }^{2} \mathrm{~J}$ Kadota. 'Department of Urology, Sapporo Medical University, Sapporo, Japan; ${ }^{2}$ The members of Urogenital Sub-committee and the Surveillance Committee of Japanese Society of Chemotherapy (JSC), Japanese Association for Infectious Diseases (JAID) and Japanese Society for Clinical Microbiology (JSCM), Tokyo, Japan; ${ }^{3}$ Research Center for Anti-Infectious Drugs, Kitasato University, Tokyo, Japan
\end{abstract}

Chlamydia trachomatis is one of the principal pathogens for nongonococcal urethritis. There have been a few studies about novel resistant strains isolated from the patients with genital chlamydial infection. However, the current common concept indicates that those were temporary and unstable strains with decreased antimicrobial susceptibility. Three societies, the Japanese Society of Chemotherapy, Japanese Association of Infectious Diseases and Japanese Society of Clinical Microbiology, performed the first national surveillance for C. trachomatis between April 2009 and October 2010. Based on the data obtained, the current situation of antimicrobial susceptibility in C. trachomatis and the results of the previous research on antimicrobial susceptibility in $M$. genitalium are discussed.

In 51 medical facilities in 8 prefectures of Japan, urethral discharge or urethral swab specimens were collected from male patients with urethritis. The specimens were sent to the Kitazato University Research Center for Anti-infectious Drugs via BD Universal Viral Transport. There, measurement of antimicrobial susceptibilities was performed according to the standard method of the Japan Society of Chemotherapy. The drugs used for antimicrobial susceptibility testing are shown below.

From 28 facilities, 207 specimens were collected and 48 specimens were positive for $C$. trachomatis by culture. Using these specimens, antimicrobial susceptibility testing could be performed for 19 strains. The MIC50, MIC90 and range $(\mu \mathrm{g} / \mathrm{ml})$ were as follows. EM: 0.06, 0.25, and 0.03 0.25: CAM: 0.008, 0.016, and 0.004 0.03; AZM: 0.125, 0.5, and 0.06 0.5; MINO: 0.5, 1, and 0.125 2; DOXY: $0.125,0.25$, and $0.03 \sim 0.5 \mu \mathrm{g} / \mathrm{ml}$; CPFX: 2 , 4, and 1 4; LVFX: 0.25 , 0.25 , and $0.125 \sim 0.5$; TFLX: $0.125,0.25$, and $0.06 \sim 0.5$; STFX: 0.06 , 0.125 , and $0.03 \sim 0.25$.

Fortunately, there were no resistant strains of C. trachomatis in this surveillance. However, the current and future situation of antimicrobial susceptibility in the pathogens of non-gonococcal urethritis will be surveyed regularly.

\section{P2.086 ORIGINS OF REPEAT INFECTIONS WITH CHLAMYDIA TRACHOMATIS (CT) AMONG HETEROSEXUAL MEN IN TWO SOUTHERN CITIES IN THE UNITED STATES}

doi:10.1136/sextrans-2013-051184.0350
P Kissinger, 'S White, ' $\mathrm{N}$ Schmidt, ${ }^{2} \mathrm{~S} N$ Taylor, ${ }^{3} \mathrm{~L}$ Mena, ${ }^{2} \mathrm{R}$ Lillis, ${ }^{1} \mathrm{~S}$ Some, ${ }^{1} \mathrm{~K}$ Defayette, ${ }^{2} \mathrm{D}$ Martin. 'Tulane University SPHTM, New Orleans, LA, United States, ${ }^{2}$ Louisiana State University Health Sciences Center, New Orleans, LA, United States, ${ }^{3}$ University of Mississippi - Department of Medicine, Jackson, MS, United States

Background Recent clinical trials have reported high repeat infection rates (12\%-14\%) following $1 \mathrm{~g}$ azithromycin. These data suggest that single-dose azithromycin may be inadequate, but high repeat infections rates could also be explained by exposure to an original or new partner or retesting before DNA clearance. The purpose of this study was to examine the origins of repeat CT infections among men.

Methods Men diagnosed with Ct by Gen-Probe Aptima Combo 2 at STD clinics in New Orleans, and Jackson, Mississippi were retested an average of 6 weeks after treatment with single-dose azithromycin. Detailed sexual behaviour histories were collected at baseline and follow-up via computer-assisted/self-administered interview and MLST genotyping was performed.

Results Of 367 men with Ct, 222 returned for a f/u visit [mean of 45 days post-baseline (s.d. 13)] and $14 / 217$ (6.5\%) were positive. Of the $14,36 \%$ reported sexual re-exposure to a baseline partner, $14 \%$ reported sexual exposure to a new partner, $7 \%$ reported sexual exposure to both, and $43 \%$ denied sexual re-exposure. Thus far MLST genotyping completed for 3 baseline-f/up positive pairs. Two pairs with the same genotype (E/39) reported sexual re-exposure to a baseline partner and the pair with a new genotype reported sexual exposure to a new partner (D/19 to C/15).

Conclusion Early repeat infection rate among men with $\mathrm{Ct}$ in this study was lower than recently reported and about half could be explained by sexual re-exposure. Rates in the other two studies may have been inflated by high re-exposure rates or premature testing using NAAT since many of the participants were tested before 3 weeks. Studies that examine repeat infections should consider reexposure/new exposure and retest when DNA clearance is assured. Our data does not support high treatment failure rates for $1 \mathrm{~g}$ azithromycin treatment of $\mathrm{Ct}$.

\section{P2.087 IN VITRO ANTIMICROBIAL SYNERGY TESTING, USING ETEST METHODOLOGY, OF NEISSERIA GONORRHOEAE FOR EVALUATION OF SUSCEPTIBILITY WHEN USING DUAL ANTIMICROBIAL THERAPY?}

doi:10.1136/sextrans-2013-051184.0351

D Golparian, R Hadad, B Hellmark, H Fredlund, M Unemo. WHO Collaborating Centre for Gonorrhoea and other STIS, Department of Laboratory Medicine, Microbiology, Örebro University Hospital, ÖREBRO, Sweden

Background Antimicrobial resistance in Neisseria gonorrhoeae is a major public health problem worldwide. Recently, the first gonococcal isolates with high-level resistance to extended-spectrum cephalosporins, including ceftriaxone, were reported and gonorrhoea may become untreatable in certain circumstances. As a response, dual antimicrobial therapy (mainly ceftriaxone+azithromycin) has been introduced in the USA and Europe. The aim of this study was to apply a method for in vitro synergy testing, using Etest methodology, of various combinations of antimicrobials, i.e. currently used or of potential interest for future dual antimicrobial therapy.

Methods The eight WHO 2008 N. gonorrhoeae reference strains and 51 clinical $N$. gonorrhoeae isolates were investigated by synergy testing using Etest of in total 15 combinations of ceftriaxone, cefixime, azithromycin, moxifloxacin, spectinomycin, and gentamicin.

Results Highest levels of synergistic and/or additive effects, without any observed antagonistic effects, were observed for the combinations cefixime+gentamicin (in total $80 \%$ of isolates), azithromycin+gentamicin (65\%), and cefixime+azithromycin (63\%). The combination of ceftriaxone+azithromycin, currently recommended in the dual antimicrobial therapy, also showed 